\title{
Reversion to Ultrafine-Grained Austenite in a Medium-Mn AHSS
}

J T Benzing ${ }^{1}$, J Bentley ${ }^{2}$, A Kwiatkowski da Silva ${ }^{3}$, L Morsdorf ${ }^{3}$, J R McBride ${ }^{4}$, D Ponge ${ }^{3}$, B Gault ${ }^{3}$, $\mathrm{J} \mathrm{Han}^{5}$, D Raabe ${ }^{3}$, J E Wittig ${ }^{1}$

1. Interdisciplinary Materials Science, Vanderbilt University, Nashville TN, USA

2. Microscopy and Microanalytical Sciences, PO Box 7103, Oak Ridge, TN, USA

3. Max-Planck-Institut für Eisenforschung, Düsseldorf, Germany

4. Vanderbilt Institute of Nanoscale Science and Engineering, Vanderbilt University, Nashville, TN, USA

5. Materials Science and Engineering, Chungnam National University, Daejeon, Republic of Korea

Third generation advanced high-strength steels (AHSS) consist of alloying contents below $17 \mathrm{wt} \%$ and multi-phase microstructures of austenite, ferrite and martensite to respectively balance cost and tensile properties [1]. The types of third generation steels capable of achieving these desired properties are medium-Mn steels, lightweight steels and quenched-and-partitioned TRIP steels [2]. Medium-Mn steels generally exhibit a fully deformed martensitic $\left(\alpha^{\prime}\right)$ microstructure after hot and cold rolling. Intercritical annealing between $\mathrm{Ac}_{1}$ and $\mathrm{Ac}_{3}$ temperatures (the $\alpha+\gamma$ phase field) and above the $\alpha^{\prime}$ recrystallization temperature produces austenite $(\gamma)$ and ferrite $(\alpha)$, which are typically ultrafine-grained (UFG) in size. The intercritical annealing temperature determines composition and thus the austenite stacking fault energy (SFE). The SFE is important for controlling transformation- and twinning-induced plasticity (TRIP/TWIP) effects that further enhance the mechanical properties of steels by increasing the strainhardening rate, even in ultrafine-grained austenite [3]. Recent work on a Fe-7Mn-0.1C-0.5Si (wt\%) steel by Kwiatkowski da Silva et al. showed that austenite reversion sequentially depends on the cosegregation of $\mathrm{C}$ and $\mathrm{Mn}$ to dislocations and grain boundaries, the formation of face-centered-cubic (FCC) $\mathrm{M}_{23} \mathrm{C}_{6}$ transition carbides, and growth of the carbides controlled by $\mathrm{C}$ diffusion and local equilibrium at the interface, culminating in the nucleation of UFG FCC austenite [4]. The present study uses scanning transmission electron microscopy energy-dispersive X-ray spectroscopy (STEM-EDS) performed using a $200 \mathrm{kV}$ FEI Tecnai Osiris equipped with a quad Super-X detector and atom probe tomography (APT) using a CAMECA LEAP $5000 \mathrm{XS}$ in laser-pulse mode (70 K and 15\% evaporation rate) to measure UFG phase compositions in a medium-Mn steel.

Intercritical annealing of a cold-rolled Fe-12Mn-3Al-0.05C (wt\%) steel was performed at $585{ }^{\circ} \mathrm{C}$. For this temperature, Thermo-Calc ${ }^{\circledR}$ simulations predict a $\mathrm{RT}$ austenite volume fraction of $44 \%$, a RT austenitic SFE of $21 \mathrm{~mJ} / \mathrm{m}^{2}$, a RT austenite composition of Fe-20.8Mn-2.1 Al-0.11C (wt\%) and a RT ferrite composition of Fe-4.9Mn-3.7Al-0.001C (wt\%). Samples were annealed for $0.5,8$ and $48 \mathrm{~h}$ at $585^{\circ} \mathrm{C}$. Electron backscatter diffraction (EBSD) measurements (viewed in the normal direction) respectively yielded an austenite volume fraction of $35 \%$ after $8 \mathrm{~h}$ at $585^{\circ} \mathrm{C}$. Samples annealed for $8 \mathrm{~h}$ (see Figure 1) revealed UFG austenite with compositions of $\sim 21 \mathrm{wt} \% \mathrm{Mn}$, (thus matching equilibrium Thermo-Calc predictions). 3D APT offers improved spatial resolution for measurements of composition profiles across UFG austenite-ferrite boundaries (see Figure 2). APT results are consistent with STEMEDS, but also reveal C-rich and C-poor boundaries. 3D FIB-EBSD measurements are underway and will be coupled with crystal plasticity finite-element modeling to investigate the effects of microstructure morphology on strain partitioning in this multi-phase medium-Mn TWIP-TRIP steel [5]. 
References:

[1] D K Matlock and J G Speer, pp. 185-205 in Microstruct. Texture Steels, ed A Halder, S Suwas and D Bhattacharjee (Springer, London), 2009.

[2] Y-K Lee and J Han, Mater. Sci. Technol. 31 (2015), p. 843.

[3] S Lee and B C De Cooman, Metall. Mater. Trans. A 44 (2013), p. 5018.

[4] A Kwiatkowski da Silva et al, Acta Mater. 147 (2018), p. 165.

[5] This work was funded by the US National Science Foundation, Division of Materials Research under grant DMR1309258, NSF EPS 1004083 and the Max-Planck-Institut für Eisenforschung in Düsseldorf, Germany.
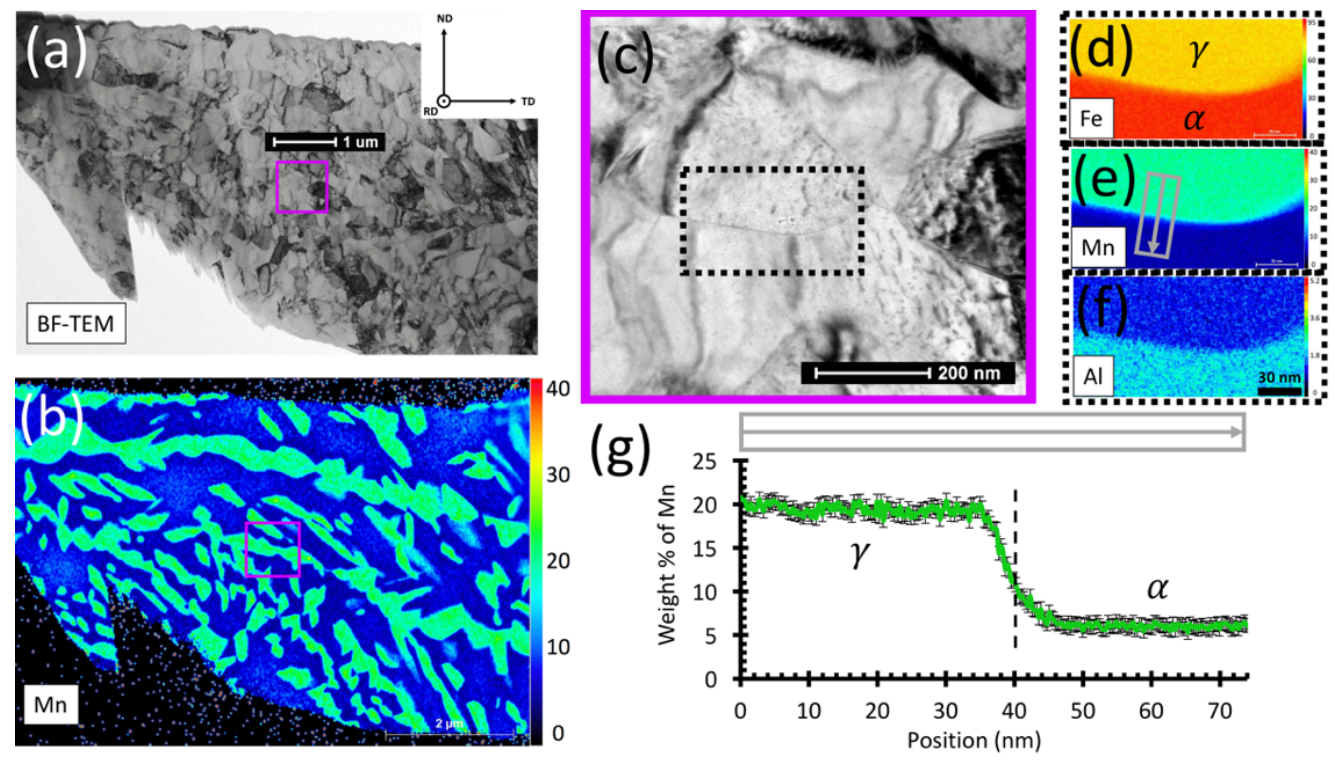

Figure 1. (a) BF-TEM image of a FIB lift-out from Fe-12Mn-3Al-0.05C (wt\%) steel (cold-rolled and annealed $8 \mathrm{~h}$ at $585^{\circ} \mathrm{C}$ ), viewed in the rolling direction (RD). (b) STEM-EDS hypermap of Mn content in $\mathrm{wt} \%$. (c) BF-TEM image shows a near edge-on boundary between UFG austenite $(\gamma)$ and UFG ferrite $(\alpha)$. Hypermaps of the marked area are provided: (d) 0-95 wt $\%$ Fe, (e) 0-40 wt $\% \mathrm{Mn}$ and (f) $0-5.2 \mathrm{wt} \%$ $\mathrm{Al}$, as well as (g) a linescan across the $\gamma$ - $\alpha$ boundary.
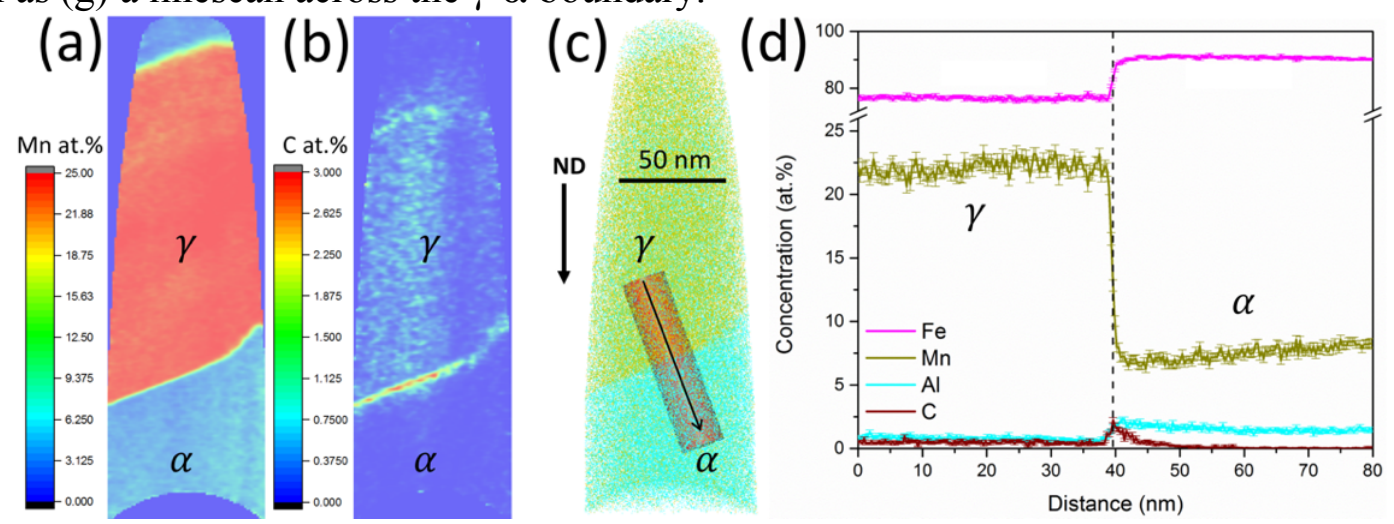

Figure 2. APT reconstruction of $150,000,000$ ions measured from a FIB-prepared needle of Fe-12Mn$3 \mathrm{Al}-0.05 \mathrm{C}\left(\mathrm{wt} \%\right.$ ) steel (cold-rolled and annealed for $8 \mathrm{~h}$ at $585^{\circ} \mathrm{C}$ ). The quantitative maps of (a) Mn and (b) $\mathrm{C}$ in at.\% measure the composition across the entire width of UFG austenite $(\gamma)$. (c) Image showing $\mathrm{Mn}$ and $\mathrm{Al}$ ions and position of the cylinder used to measure (d) a profile across the $\gamma$ - $\alpha$ boundary. 